\title{
A Recording Photoelastic Stress Meter
}

\author{
Authors report on the development of an instrument for \\ recording transient stresses at a point. A specific \\ application is described
}

by R. Gardon, R. W. Bayma and A. Warnick

\begin{abstract}
An instrument for automatically making photoelastic measurements of stress is described. It may be used for recording transient stresses at a point or for scanning spatial stress distributions. Its use is illustrated by an application entailing the measurement of transient thermal stresses in a viscoelastic material.
\end{abstract}

\section{Introduction}

When applied to the measurement of transient stresses, conventional photoelastic methods have certain drawbacks. The most general approach,

R. Gardon and A. Warnick are Principal Research Engineer and Principal Research Scientist, Scientific Research Staff, Ford Motor Co., Dearborn, Mich. R.W. Bayma is Graduate Student, Information and Control Engineering Program, University of Michigan, Ann Arbor, Mich.

Paper was presented at 1966 SESA Spring Meeting held in Detroit, Mich., on May 4-6. that of taking moving pictures of instantaneous fringe patterns, entails rather laborious interpretation. ${ }^{1,2}$ Where, as is often the case, the temporal variation of stresses is required to be known at one point only - so that one would actually use only a small fraction of the information contained in the pictures-one can resort to simpler techniques. The counting of fringes as they pass the point in question is one. However, it lacks resolution, especially where the total number of fringes is small. The use of a conventional Babinet or similar compensator is often ruled out by the inability of an operator to follow rapidly changing stresses. ${ }^{3}$

This paper reports on an instrument for automatically recording transient stresses at one point, which is much simpler to use than the usual cine-

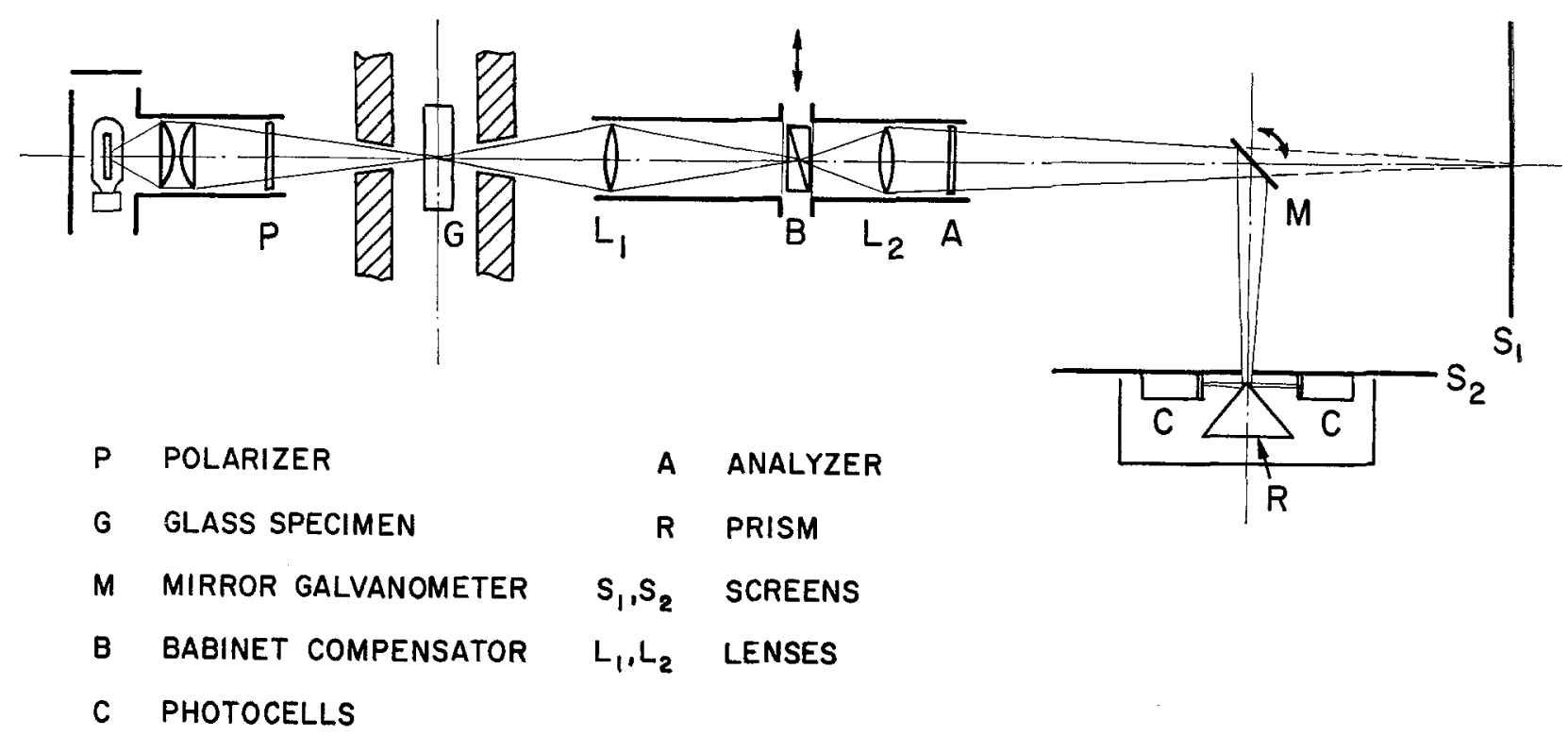

Fig. 1-Optical system of recording stress meter 
matographic technique, has a better resolution than the counting of fringes and a much faster response than could be achieved with a conventional, manually operated compensator. Though conceived for the measurement of transient stresses, the instrument may also lend itself to applications such as the automatic scanning of spatially varying stress distributions as, for example, in finished glass parts on a production line.

Since the present development had its roots in work with glass, the recording stress meter will be described with an optical system suitable for transparent specimens. However, its principle should be equally applicable to other optical systems, such as those involving photoelastic coatings and reflection optics.

\section{Description of Apparatus}

The optical system of the recording stress meteras set up for studies of the tempering and annealing of glass-is shown in Fig. 1. A collimated beam of light, polarized at $45 \mathrm{deg}$ to the axis of a glass specimen $G$, is passed edgewise through the specimen. For annealing, this is located in a furnace having two small, diametrically located windows. Lens $L_{1}$ forms an image of the edge of the specimen in the

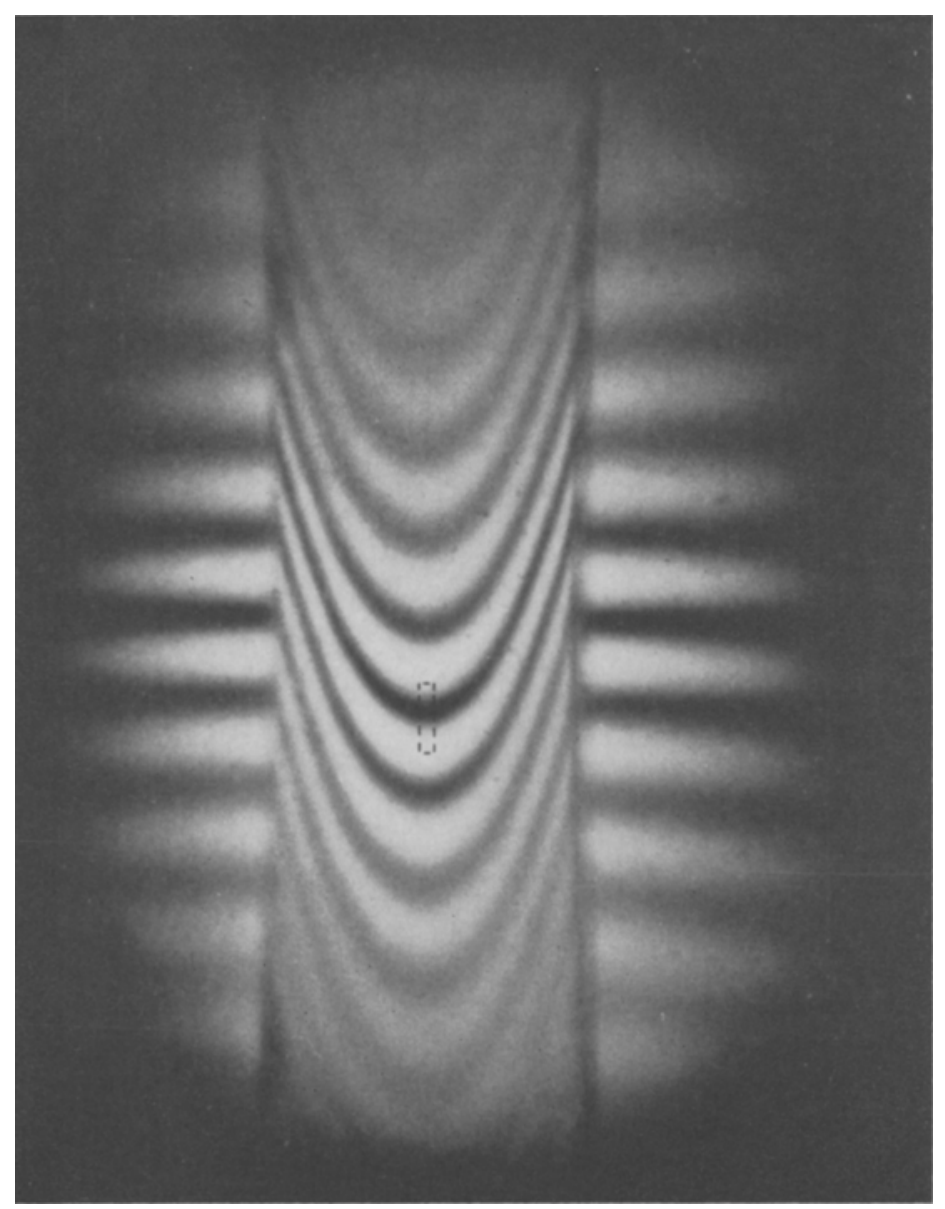

Fig. 2-Stress distribution in mildly tempered glass. Small rectangle represents that part of image admitted to the fringe scanner plane of the Babinet compensator $B$, while a second lens $L_{2}$ images both the specimen and the Babinet on a screen $S_{1}$. With the interposition of the analyzer $A$, a pattern of fringes is projected on the screen. The pattern produced by the Babinet alone is a system of parallel straight lines at right angles to the specimen. A parabolic stress distribution in the specimen then produces corresponding parabolic fringes, as shown in Fig. 2.

So far we have described a conventional photoelastic system with a compensator. We now add a small mirror $M$, which is mounted on a galvanometer. It is used to reflect the image of the fringes onto a second screen $S_{2}$, in which there is a short slit to isolate a narrow beam of light that has passed through the mid-plane of the specimen. This beam is allowed to fall on a right-angled prism $R$, which serves as a beam-splitter and divides the light between two matched photocells $C$. These are differentially connected and serve as the sensing element of an electronic control system which supplies current to the galvanometer and, thereby, positions the mirror in such a manner as to maintain a selected point of a selected fringe centered on the apex of the beam splitter. Thus, a change of the local stress in the specimen (or a movement of the Babinet) is translated into a small compensatory rotation of the mirror-galvanometer, and the current used to produce this becomes a measure of the change in optical retardation. This use of a galvanometer-instead of some servomotor to reposition the Babinet-makes for a very simple and inexpensive system. The required dynamic response and steady-state accuracy are obtained by integrating the d-c error signal from the photocells with an operational amplifier. The circuit diagram is shown in Fig. 3.

Before using the instrument, the selected fringe must be aligned with the photocells. To do this, the Mode Selector (cf. Fig. 3) is switched to Reset, which also allows the mirror to be rotated at will by means of the Initial Position Control. The initial setting of the instrument can thus also be matched to any non-zero initial stress in the specimen. The adjustment need only be approximate, since in the Operate mode the fringe scanner instantly locks onto the nearest fringe. Henceforth, any change in stress (and corresponding displacement of the fringe) is continuously compensated for by rotation of the galvanometer-mirror, and the required galvanometer current is recorded. Finally, the Automatic Reset mode permits remote and automatic switching from Operate to Reset and back as successive specimens are introduced into the optical system.

It may be of interest to note that the slit in screen $S_{2}$ that admits light to the fringe scanner is 0.050 -in. wide, corresponding to about $1 / 20$ of the (magnified) image of the edge of the specimen.* To maximize the optical gain of the scanning sys-

* The part of the image admitted to the fringe scanner is outlined in Fig. 2. 


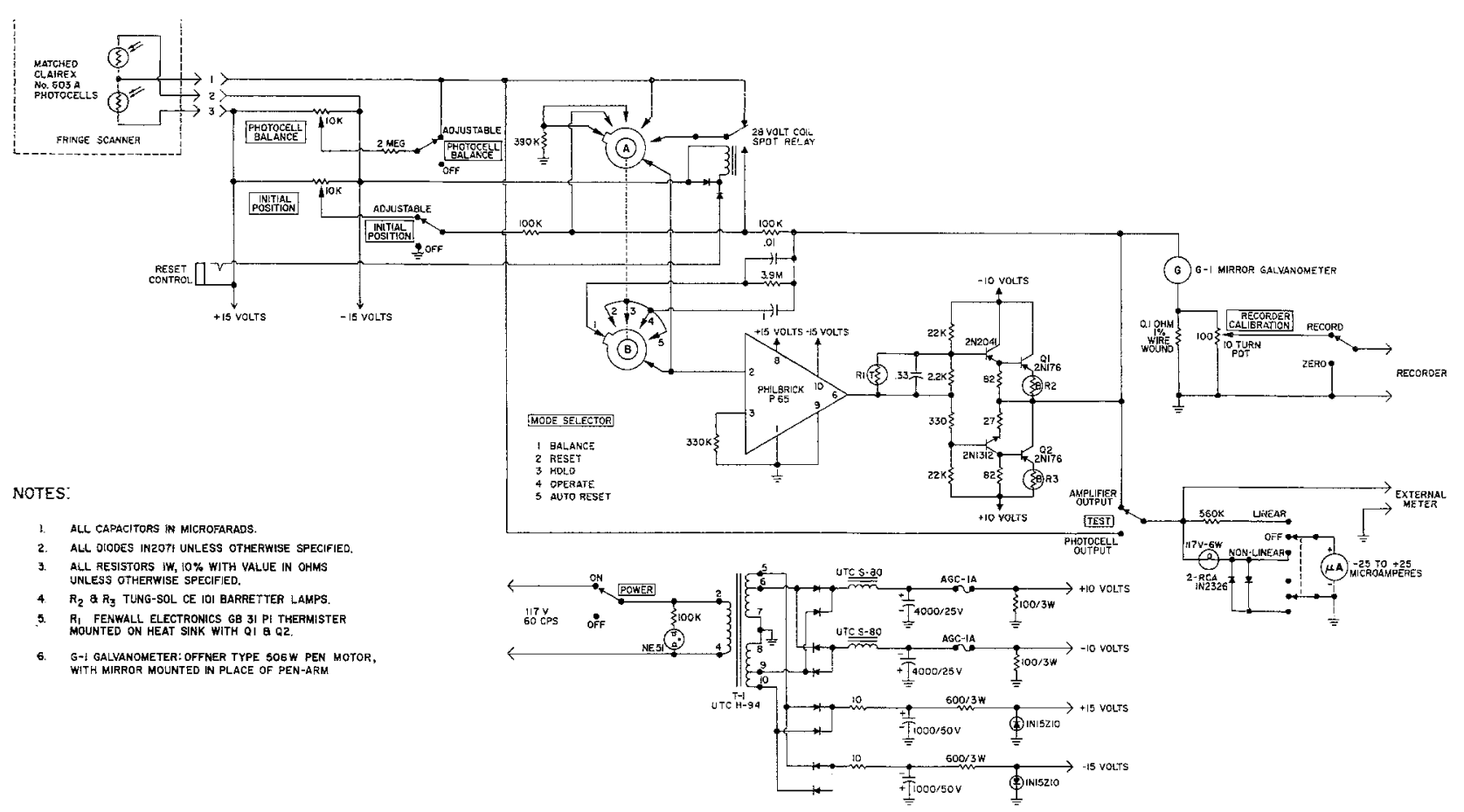

Fig. 3-Circuit diagram of recording stress meter

tem, the length of the slit (1/4 in.) is about equal to one fringe spacing. The photocells used are Clairex type 603-A cadmium-selenide photoconductive cells, chosen for their small size, sensitivity and relatively rapid response. At the level of illumination used, they have a time constant of approximately 10 msec. Their peak spectral response is in the near infrared range at $7350 \AA$. Because of their low response to fluorescent lighting and their differential connection, the instrument can be used with normal room illumination, and its operation is unaffected by changes in this illumination. Since only one fringe is involved in the operation of the instrument, there is no particular advantage to using monochromatic light. Indeed, it is more convenient to use white light, which makes identification of the fringes easier. The black, zeroth-order fringe is usually used, though the fringe scanner will also operate locked onto some of the other low-order fringes.

\section{Performance}

The present instrument was designed to measure stress-induced optical retardation over a range of $7000 \mathrm{~m} \mu . f^{\dagger}$ Its zero is arbitrarily adjustable by the position of the Babinet, so that it can be used from, say, $-2000 \mathrm{~m} \mu$ (compression) to $+5000 \mathrm{~m} \mu$ (tension). The total range is determined by the permissible rotation of the galvanometer, the fringe spacing of the Babinet compensator and its magnification by the lens $L_{2}$. It can readily be in-

†The $m \mu$ (millimicron) is used here as the unit of retardation, whichunlike the number of fringes- is independent of the wavelength of light used. In terms of the sodium $D$ line, one fringe spacing corresponds to $589 \mathrm{m \mu}$, and the range of the instrument is therefore approximately 12 fringe orders. creased by using a Babinet with a closer spacing of fringes.

The instrument is being used in conjunction with a 10-mv full-scale recorder. By means of the adjustments provided, the calibration can be set to whatever scale of stress may be convenient, say 500 $\mathrm{m} \mu$ retardation/in. of chart paper. Basically the electrical output of the device is a linear function of the fringe displacement; however, some nonlinearity is introduced into the over-all system by the inexpensive optical elements used (cf. ordinate scale of Fig. 4).

Calibration is accomplished by making the fringe scanner follow mechanical displacements of the Babinet compensator, the position of which is precisely adjustable by means of a micrometer.

A slight hysteresis, probably of mechanical origin, limits the reproducibility of readings to about 10 $\mathrm{m} \mu$, which represents 0.15 percent of the range of the instrument. The accuracy of recordings is limited mainly by the movement or vibration of the specimen and is, of course, best where stress gradients are small, so that slight lateral displacements of the specimen have little effect on the stress "seen" by the instrument.

The largest step-function to which the instrument may be subjected is one-half of a fringe spacing. Its response to a small step function, as for example during the switching from an imperfectly adjusted Reset to Operation, is practically instantaneous. I The instrument was designed to follow a ramp input of a 2 fringes/sec with a steady-state error of $1 / 50$

\$ Such a "step" can advantageously be used as a synchronization mark, as shown in Fig. 4. 
fringe. i.e., about $10 \mathrm{~m} \mu$. It can, therefore, follow even the most rapid stress changes to which it is being subjected with negligible lag. Its natural frequency is about $15 \mathrm{cps}$.

\section{An Application}

Figure 4 illustrates some results obtained in the application of the recording stress meter to a study of the tempering of glass plates.

Tempering entails heating the glass to a temperature high enough for stresses in it to relax. The hot, substantially stress-free glass is then subjected to intense quenching, which rapidly cools it through the temperature range in which glass is viscoelastic. The interplay of transient thermal stresses and (partial) stress relaxation while the glass is still hot produces permanent stresses, "frozen" into the glass, which serve to strengthen it by compressively prestressing its surfaces. The stress distribution across the thickness of the glass is parabolic, the surface compression being balanced by internal tension. This tension is greatest in the mid-plane of the glass and is commonly used as a measure of the degree of temper. With the present apparatus, we can measure not only its final value but also its evolution during the quenching process.

For purposes of comparison, curve (A) of Fig. 4 shows the temporal variation of (calculated) thermal stresses in the mid-plane of an elastic plate during quenching from a uniform, high temperature. These stresses would be compressive throughout. They would increase from an initial zero to a maximum at the instant temperature gradients through the glass are at their maximum, and return to zero as the plate returns to isothermal conditions at room temperature.

Curves (B) and (C) are recordings of two characteristically different stress-time histories in glass plates undergoing tempering. $\S$ Curve (B) was recorded with glass initially hot enough for viscous stress relaxation to be practically instantaneous, so that no thermal stresses arose during the early stages of quenching, while temperature gradients in the glass were increasing. "Solidification" of the glass occurred only after temperature gradients had begun to decrease again. This evoked a tensile stress in the mid-plane, which increased monotonically to a final, permanent value approximately as large as the maximum temporary compression shown by curve (A). Curve (C) refers to glass quenched from a lower temperature, so that it solidified earlier in the process, while temperature gradients were still increasing. This undesirable

\$n Fig. 4, stresses are recorded in terms of the stress-induced retardation, $R$, in $m \mu$. To convert this into conventional units of stress, the following equation is used:

$$
\left(P_{1}-P_{2}\right)=\frac{R}{L} \times B
$$

where $P_{1}$ and $P_{2}$ are the two principal stresses ( $p$ si) (in the present case $\left.P_{2}=0\right), L$ is the optical path length (in.), and $B$ the stress-optical coefficient $[\mathrm{psi} /(\mathrm{m \mu} / \mathrm{in.})]$ (cf. Ref. 3$)$.

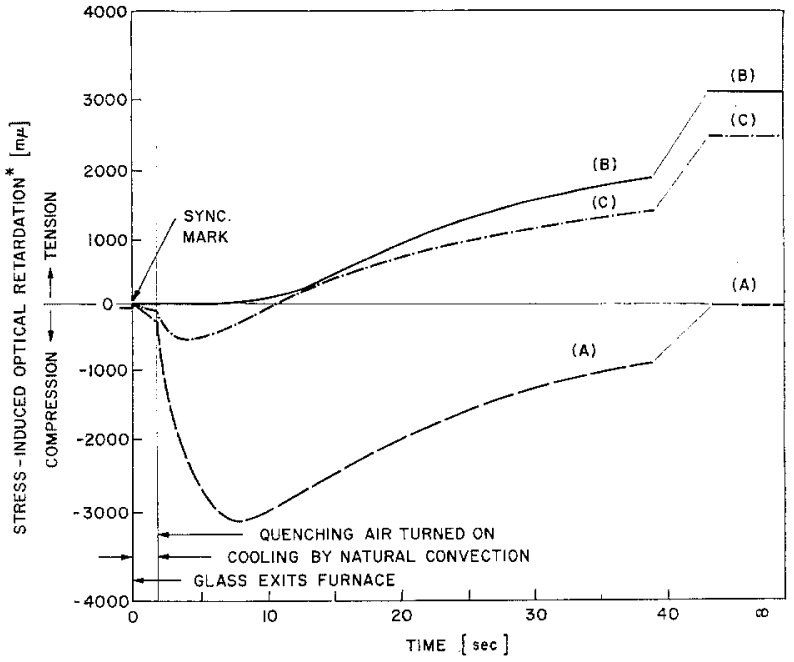

\begin{tabular}{|c|l|c|c|}
\hline Curve & & $\begin{array}{c}\text { Initial } \\
\text { Temp. } \\
{ }^{\circ} \mathrm{C}\end{array}$ & $\begin{array}{c}\text { Permanerit } \\
\text { Retardation } \\
\mathrm{m}^{*}\end{array}$ \\
\hline (A) & $\begin{array}{l}\text { Calculated for Glass - Like } \\
\text { Elastic Moterial }\end{array}$ & 680 & 0 \\
(B) & Experimental for Glass & 743 & 3070 \\
(C) & Experimental for Glass & 613 & 2540 \\
\hline
\end{tabular}

Glass Thickness $\quad=0.61 \mathrm{~cm}$ Quenching Coefficient $=0.0053 \mathrm{col} / \mathrm{cm}^{2} .{ }^{\circ} \mathrm{C} \cdot \mathrm{sec}$ * Optical Path Length $=2.46 \mathrm{~cm}$ Retardation of $1000 \mathrm{~m} \mu$ ther

Fig. 4-Stresses in the mid-plane of glass plates during tempering

condition resulted in some temporary compression of the mid-plane of the glass -as with the elastic plate. With time, this compression diminished again, partly on account of stress relaxation, partly on account of the reversal from increasing to decreasing temperature gradients. Ultimately, the effect of the latter predominated, again bringing into being a permanent-though somewhat reduced - tensile stress in the mid-plane of the glass.

A fuller account of the stress-time-position history of glass undergoing tempering is given in Ref. 2 . It is complicated by the fact that, during the initial stages of quenching, the stress distributions are not parabolic and change in shape as well as magnitude. Figure 7 of Ref. 2 clearly shows this with the aid of selected frames of cinematographic records of various tempering treatments. Their further discussion is beyond our present scope. Suffice it to note that, where a full account of stresses at all points is not required, the direct recording of stress-time histories at selected points is much the simpler procedure.

\section{References}

1. Acloque, P., "Étude expérimentale de l'effet de certains traitements thermiques sur le verre el de leur influence sur les contraintes mécaniques internes," Verres et Refractaries, 1, 10-19 (February 1950).

2. Gardon, R., "The Tempering of Flat Glass by Forced Convection," Paper No. 79, Proc. 7th Intl. Congr, on Glass, Bruxelles (1965).

3. VanZee, A. F., and Noritake, H. M., "Measurement of Stress-Optical Coefficient and Rate of Stress Release in Commercial Soda-Lime Glasses," Jnl. Am. Ceramic Soc., 41, 164-175 (1958). 